FACTA UNIVERSITATIS (NIŠ)

Ser. Math. Inform. Vol. 35, No 2 (2020), 333-349

https://doi.org/10.22190/FUMI2002333Y

\title{
CLASSIFICATION OF CONFORMAL SURFACES OF REVOLUTION IN HYPERBOLIC 3-SPACE
}

\author{
Nural Yuksel and Murat Kemal Karacan
}

(C) 2020 by University of Niš, Serbia | Creative Commons Licence: CC BY-NC-ND

Abstract. In this paper, we classify conformal surfaces of revolution in hyperbolic 3 -space $\mathbb{H}^{3}\left(-c^{2}\right)$ satisfying an equation in terms of the position vector field and the Laplace operators with respect to the first, the second and the third fundamental forms of the surface.

Keywords: hyperbolic 3-space; Laplace operators; fundamental forms of the surface.

\section{Introduction}

Surfaces of revolution is form of the most easily recognized class of surfaces. The use of surfaces of revolution is essential in many fields such as physics and engineering. Surfaces of revolution have been well known since ancient times as well as common objects in geometric modelling which can be found everywhere in nature, human artefacts, technical practice and also in mathematics. Furthermore, many objects from everyday life such as cans, table glasses and furniture legs are surfaces of revolution. The process of lathing wood produces surfaces of revolution by its very nature $[1,19]$.

The notion of finite type immersion of submanifolds of a Euclidean space has been used in classifying and characterizing the well known Riemannian submanifolds. Chen posed the problem of classifying the finite type surfaces in the 3dimensional Euclidean space $\mathbb{E}^{3}$. A Euclidean submanifold is said to be of Chen finite type if its coordinate functions are a finite sum of eigenfunctions of its Laplacian $\Delta$. Further, the notion of finite type can be extended to any smooth function on a submanifold of a Euclidean space or a pseudo-Euclidean space. The theory of submanifolds of finite type has been studied by many geometers [7, 11].

In $\mathbb{H}^{3}\left(-c^{2}\right)$, surfaces of constant mean curvature $\mathbf{H}=c$ are particularly interesting, because they exhibit many geometric properties in common with minimal

Received April 17, 2019; accepted October 31, 2019

2010 Mathematics Subject Classification. Primary 53A10; Secondary 53A35, 53C50 
surfaces in $\mathbb{E}^{3}$. This is not a coincidence. There is a one-to-one correspondence, socalled Lawson correspondence, between surfaces of constant mean curvature $\mathbf{H}_{h}$ in $\mathbb{H}^{3}\left(-c^{2}\right)$ and surfaces of constant mean curvature $\mathbf{H}_{e}=\sqrt{\mathbf{H}_{h}^{2}-c^{2}}$. Those corresponding constant mean curvature surfaces satisfy the same Gauss-Codazzi equations, so they share many geometric properties in common. Lee and Zarske constructed surfaces of revolution with constant mean curvature $\mathbf{H}=c$ and minimal surfaces of revolution in hyperbolic 3 -space $\mathbb{H}^{3}\left(-c^{2}\right)$ of constant curvature $-c^{2}$. In addition, they have showed that, the limit of the surfaces of revolution with $\mathbf{H}=c$ in $\mathbb{H}^{3}\left(-c^{2}\right)$ is a catenoid, the minimal surface of revolution in Euclidean 3space as $c$ approaches 015 . Lee and Martin studied spacelike and timelike surfaces of constant mean curvature in de sitter 3 -space $[14,16]$. Kaimakamis, Papantoniou and Petoumenos studied Lorentz invariant spacelike surfaces of constant mean curvature in anti de sitter 3 -space [12].

We know that, $\mathbf{x}$ is harmonic if $\Delta \mathbf{x}=0$ in Euclidean 3-space. However, this is no longer true in $\mathbb{H}^{3}\left(-c^{2}\right)$ because the Laplacian equation $\Delta \mathbf{x}=0$ is not the harmonic map equation in $\mathbb{H}^{3}\left(-c^{2}\right)$ [15].

Let $\mathbf{x}: \mathbf{M} \rightarrow \mathbb{E}^{m}$ be an isometric immersion of a connected $n$-dimensional manifold in the $m$-dimensional Euclidean space $\mathbb{E}^{m}$. Denote by $\mathbf{H}$ and $\Delta$ the mean curvature and the Laplacian of $\mathbf{M}$ with respect to the Riemannian metric on $\mathbf{M}$ induced from that of $\mathbb{E}^{m}$, respectively [6]. Takahashi proved that the submanifolds in $\mathbb{E}^{m}$ satisfying $\Delta x=\lambda x$, that is, all coordinate functions are eigenfunctions of the Laplacian with the same eigenvalue $\lambda \in \mathbb{R}$ are either the minimal submanifolds of $\mathbb{E}^{m}$ or the minimal submanifolds of hypersphere $\mathbb{S}^{m-1}$ in $\mathbb{E}^{m}[18]$.

As an extension of Takahashi theorem, Garay studied hypersurfaces in $\mathbb{E}^{m}$ whose coordinate functions are eigenfunctions of the Laplacian, but not necessarily associated to the same eigenvalue. He considered hypersurfaces in $\mathbb{E}^{m}$ satisfying the condition

$$
\Delta x=A x,
$$

where $A \in M a t(m, \mathbb{R})$ is an $m \times m$ - diagonal matrix, and proved that such hypersurfaces are minimal $(\mathbf{H}=0)$ in $\mathbb{E}^{m}$ and open pieces of either round hyperspheres or generalized right spherical cylinders [10].

Related to this, Dillan, Pas and Vertraelen investigated surfaces in $\mathbb{E}^{3}$ whose immersions satisfy the condition

$$
\Delta x=A x+B,
$$

where $A \in \operatorname{Mat}(3, \mathbb{R})$ is a $3 \times 3$-real matrix and $B \in \mathbb{R}^{3}[8]$. In other words, each coordinate function is of 1-type in the sense of Chen [7]. For the Lorentzian version of surfaces satisfying (1.2), Alias, Ferrandez and Lucas proved that the only such surfaces are minimal surfaces and open pieces of Lorentz circular cylinders, hyperbolic cylinders, Lorentz hyperbolic cylinders, hyperbolic spaces or pseudo-spheres [2].

The notion of an isometric immersion $\mathbf{x}$ is naturally extended to smooth functions on submanifolds of Euclidean space or pseudo-Euclidean space. The most 
natural one of them is the Gauss map of the submanifold. In particular, if the submanifold is a hypersurface, then the Gauss map can be identified with the unit normal vector field to it. Dillen and Vertraelen studied surfaces of revolution in the three dimensional Euclidean space $\mathbb{E}^{3}$ such that its Gauss map $G$ satisfies the condition

$$
\Delta G=A G,
$$

where $A \in$ Mat $(3, \mathbb{R})[9]$. Baikoussis and Vertraelen studied the helicoidal surfaces in $\mathbb{E}^{3}[3]$. Choi completely classified the surfaces of revolution satisfying the condition (1.3) in the three dimensional Minkowski space $\mathbb{E}_{1}^{3}[5]$. Bekkar, Zoubir and Senoussi classified surfaces of revolution satisfying (1.1) in the three dimensional Minkowski space $[4,17]$. Kaimakamis, Papantpniou and Peteoumenos classified surfaces of revolution satisfying

$$
\Delta^{I I I} r=A r,
$$

in the three dimensional Lorentz-Minkowski space [12]. Choi, Kim and Yoon investigated the surfaces of revolution satisfying an equation in terms of the position vector field and the 2nd-Laplacian in Minkowski 3-space [6].

The main purpose of this paper is complete the classification of conformal surfaces of revolution in $\mathbb{H}^{3}\left(-c^{2}\right)$ in terms of the position vector field and the Laplacian operators.

\section{Preliminaries}

Let $\mathbb{R}^{3+1}$ denote the Minkowski spacetime with rectangular coordinates $x_{0}, x_{1}, x_{2}, x_{3}$ and the Lorentzian metric

$$
d s^{2}=-\left(d x_{0}\right)^{2}+\left(d x_{1}\right)^{2}+\left(d x_{2}\right)^{2}+\left(d x_{3}\right)^{2} .
$$

Hyperbolic 3-space is the hyperquadric:

$$
\mathbb{H}^{3}\left(-c^{2}\right)=\left\{\left(x_{0}, x_{1}, x_{2}, x_{3}\right) \in \mathbb{R}^{3+1} \mid-x_{0}^{2}+x_{1}^{2}+x_{2}^{2}+x_{3}^{2}=-\frac{1}{c^{2}}\right\}
$$

which has the constant sectional curvature $-c^{2}$. This is a hyperboloid of two sheets in spacetime so it is called the hyperboloid model of hyperbolic 3 -space. Consider the chart

$$
U=\left\{\left(x_{0}, x_{1}, x_{2}, x_{3}\right) \in \mathbb{H}^{3}\left(-c^{2}\right) \mid \quad x_{0}+x_{1}>0\right\}
$$

and define

$$
\begin{gathered}
t=-\frac{1}{c} \log c\left(x_{0}+x_{1}\right), \\
x=\frac{x_{2}}{c\left(x_{0}+x_{1}\right)},
\end{gathered}
$$




$$
y=\frac{x_{3}}{c\left(x_{0}+x_{1}\right)} .
$$

Then

$$
d s^{2}=(d t)^{2}+e^{-2 c t}\left\{(d x)^{2}+(d y)^{2}\right\} .
$$

$\mathbb{R}^{3}$ with coordinates $t, x, y$ and the metric

$$
g_{c}=(d t)^{2}+e^{-2 c t}\left\{(d x)^{2}+(d y)^{2}\right\}
$$

is called the flat model of hyperbolic 3 -space. We will still denote it by $\mathbb{H}^{3}\left(-c^{2}\right)$. The flat chart model is a local chart of hyperbolic 3-space, so it is not regarded as a standard model of hyperbolic 3 -space. As $c \rightarrow 0, \mathbb{H}^{3}\left(-c^{2}\right)$ flattens out to Euclidean 3 -space $\mathbb{E}^{3}[15]$.

Let $\mathbb{R}^{3}$ be equipped with the metric

$$
d s^{2}=(d t)^{2}+e^{-2 c t}\left\{(d x)^{2}+(d y)^{2}\right\} .
$$

The space $\left(\mathbb{R}^{3}, g\right)$ has constant curvature $-c^{2}$. It is denoted by $\mathbb{H}^{3}\left(-c^{2}\right)$ and is called the pseudospherical model of hyperbolic 3 -space. From the metric (2.1), one can easily see that $\mathbb{H}^{3}\left(-c^{2}\right)$ flattens out to $\mathbb{E}^{3}$ Euclidean 3-space as $c \rightarrow 0[15]$.

Let $\mathbf{M}$ be a domain and $\mathbf{x}: \mathbf{M} \rightarrow \mathbb{H}^{3}\left(-c^{2}\right)$ a parametric surface. The metric (2.1) induces an inner product on each tangent space $T_{p} \mathbb{H}^{3}\left(-c^{2}\right)$. This inner product can be used to define conformal surfaces in $\mathbb{H}^{3}\left(-c^{2}\right) . \mathbf{x}: \mathbf{M} \rightarrow \mathbb{H}^{3}\left(-c^{2}\right)$ is said to be conformal if

$$
\left\langle\mathbf{x}_{u}, \mathbf{x}_{v}\right\rangle=0 \quad\left|\mathbf{x}_{u}\right|=\left|\mathbf{x}_{v}\right|=e^{\frac{w}{2}},
$$

where $(u, v)$ is a local coordinate system in $\mathbf{M}$ and $w: \mathbf{M} \rightarrow \mathbb{R}$ is a real-valued function in $\mathbf{M}$. The induced metric on the conformal parametric surface is given by

$$
d s_{\mathbf{x}}^{2}=e^{w}\left((d u)^{2}+(d v)^{2}\right) .
$$

In order to calculate the mean curvature of $\mathbf{x}$, we need to find a unit normal vector field $\mathbf{G}$ of $\mathbf{x}$. For that, we need something like cross product. $\mathbb{H}^{3}\left(-c^{2}\right)$ is not a vector space but we can define an analogue of cross product locally on each tangent space $T_{p} \mathbb{H}^{3}\left(-c^{2}\right)$. Let

$$
\begin{aligned}
\mathbf{v} & =v_{1}\left(\frac{\partial}{\partial t}\right)_{p}+v_{2}\left(\frac{\partial}{\partial x}\right)_{p}+v_{3}\left(\frac{\partial}{\partial y}\right)_{p}, \\
\mathbf{w} & =w_{1}\left(\frac{\partial}{\partial t}\right)_{p}+w_{2}\left(\frac{\partial}{\partial x}\right)_{p}+w_{3}\left(\frac{\partial}{\partial y}\right)_{p},
\end{aligned}
$$

$x, y \in T_{p} \mathbb{H}^{3}\left(-c^{2}\right)$, where $\left\{\left(\frac{\partial}{\partial t}\right)_{p},\left(\frac{\partial}{\partial x}\right)_{p},\left(\frac{\partial}{\partial y}\right)_{p}\right\}$ denote the canonical basis for $T_{p}$ $\mathbb{H}^{3}\left(-c^{2}\right)$. The cross product is defined by

$$
\mathbf{v} \times \mathbf{w}=\left(v_{2} w_{3}-v_{3} w_{2}\right)\left(\frac{\partial}{\partial t}\right)_{p}
$$




$$
\begin{aligned}
& +e^{2 c t}\left(v_{3} w_{1}-v_{1} w_{3}\right)\left(\frac{\partial}{\partial x}\right)_{p} \\
& +e^{2 c t}\left(v_{1} w_{2}-v_{2} w_{1}\right)\left(\frac{\partial}{\partial y}\right)_{p},
\end{aligned}
$$

where $p=(t ; x ; y) \in \mathbb{H}^{3}\left(-c^{2}\right)$. Then by a direct calculation we obtain

$$
g_{11}=\left\langle\mathbf{x}_{u}, \mathbf{x}_{u}\right\rangle, g_{22}=\left\langle\mathbf{x}_{v}, \mathbf{x}_{v}\right\rangle, g_{12}=\left\langle\mathbf{x}_{u}, \mathbf{x}_{v}\right\rangle .
$$

Let $\mathbf{x}: \mathbf{M} \rightarrow \mathbb{H}^{3}\left(-c^{2}\right)$ be a parametric surface. Then on each tangent plane $T_{p}$ $\mathbf{x}(\mathbf{M})$, we have

$$
\left\|\mathbf{x}_{u} \times \mathbf{x}_{v}\right\|^{2}=e^{4 c t(u, v)}\left(g_{11} g_{22}-g_{12}^{2}\right)
$$

where $p=(t(u ; v), x(u ; v), y(u ; v)) \in \mathbb{H}^{3}\left(-c^{2}\right)$. If $c \rightarrow 0,(2.3)$ becomes the familiar formula

$$
\left\|\mathbf{x}_{u} \times \mathbf{x}_{v}\right\|^{2}=g_{11} g_{22}-g_{12}^{2}
$$

from the Euclidean case. In this case, the Gaussian curvature and the mean curvature of a parametric surface $\mathbf{x}(u, v)$ may be calculated by

$$
\left\{\begin{array}{c}
\mathbf{K}=\widetilde{\mathbf{K}}+\epsilon \frac{h_{11} h_{22}-h_{12}^{2}}{g_{11} g_{22}-g_{12}^{2}} \\
\mathbf{H}=\frac{g_{22} h_{11}+g_{11} h_{22}-2 g_{12} h_{12}}{2\left(g_{11} g_{22}-g_{12}^{2}\right)}
\end{array}\right.
$$

where $\widetilde{\mathbf{K}}$ is the sectional curvature and $\mathbf{G}$ is unit normal vector field of $\mathbf{M}$, respectively. So the coefficient of second fundamental forms are given by

$$
h_{11}=\left\langle\mathbf{x}_{u u}, \mathbf{G}\right\rangle, h_{22}=\left\langle\mathbf{x}_{v v}, \mathbf{G}\right\rangle, h_{12}=\left\langle\mathbf{x}_{u v}, \mathbf{G}\right\rangle .
$$

Let $\mathbf{x}: \mathbf{M} \rightarrow \mathbb{H}^{3}\left(-c^{2}\right)$ be a conformal surface satisfying (2.2). The mean curvature $\mathbf{H}$ of $\mathbf{x}$ is given by

$$
\mathbf{H}=\frac{1}{2} e^{-w}\langle\Delta \mathbf{x}, \mathbf{G}\rangle
$$

One can easily see that the the formulas (2.4) and (2.5) coincide for conformal surfaces in $\mathbb{H}^{3}\left(-c^{2}\right)[15]$.

Rotations about the $t$-axis are the only type of Euclidean rotations that can be considered in $\mathbb{H}^{3}\left(-c^{2}\right)$. Consider a profile curve $\alpha(u)=(u, f(u), 0)$ in the $t x$-plane. Denote $\mathbf{x}(u, v)$ as the rotation of $\alpha(u)$ about the $t$-axis through an angle $v$. Then,

$$
\mathbf{x}(u, v)=(u, f(u) \cos v, f(u) \sin v) .
$$

[15]. It is well known in terms of local coordinates $\{u, v\}$ of $\mathbf{M}$ the Laplacian operators $\Delta^{\mathbf{I}}, \Delta^{\mathbf{I I}}, \Delta^{\mathbf{I I I}}$ of the first, the second and the third fundamental forms on $\mathbf{M}$ are defined by $[4,12,17]$

$$
\Delta^{\mathbf{I}} \mathbf{x}=-\frac{1}{\sqrt{\left|g_{11} g_{22}-g_{12}^{2}\right|}}\left[\frac{\partial}{\partial u}\left(\frac{g_{22} \mathbf{x}_{u}-g_{12} \mathbf{x}_{v}}{\sqrt{\left|g_{11} g_{22}-g_{12}^{2}\right|}}\right)-\frac{\partial}{\partial v}\left(\frac{g_{12} \mathbf{x}_{u}-g_{11} \mathbf{x}_{v}}{\sqrt{\left|g_{11} g_{22}-g_{12}^{2}\right|}}\right)\right],
$$




$$
\Delta^{\mathbf{I I}} \mathbf{x}=-\frac{1}{\sqrt{\left|h_{11} h_{22}-h_{12}^{2}\right|}}\left[\frac{\partial}{\partial u}\left(\frac{h_{22} \mathbf{x}_{u}-h_{12} \mathbf{x}_{v}}{\sqrt{\left|h_{11} h_{22}-h_{12}^{2}\right|}}\right)-\frac{\partial}{\partial v}\left(\frac{h_{12} \mathbf{x}_{u}-h_{11} \mathbf{x}_{v}}{\sqrt{\left|h_{11} h_{22}-h_{12}^{2}\right|}}\right)\right]
$$

and

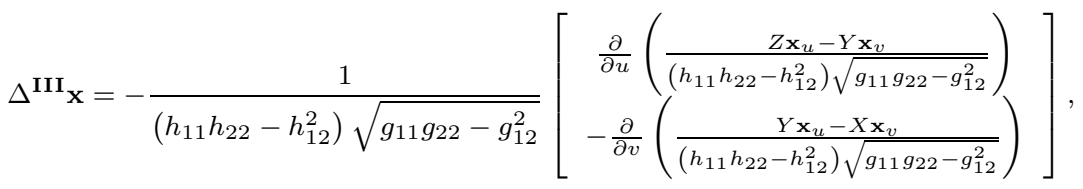

where

$$
\begin{aligned}
X & =g_{11} h_{12}^{2}-2 g_{12} h_{11} h_{12}+g_{22} h_{11}^{2}, \\
Y & =g_{11} h_{12} h_{22}-g_{12} h_{11} h_{22}+g_{22} h_{11} h_{12}-g_{12} h_{12}^{2}, \\
Z & =g_{22} h_{12}^{2}-2 g_{12} h_{22} h_{12}+g_{11} h_{22}^{2} .
\end{aligned}
$$

\section{Conformal Surfaces of Revolution Satisfying $\Delta^{\mathrm{I}} \mathbf{x}=\mathbf{A x}$}

In this section, we will classify conformal surfaces of revolution $\mathbb{H}^{3}\left(-c^{2}\right)$ satisfying the equation

$$
\Delta^{\mathrm{I}} \mathbf{x}=\mathbf{A x}
$$

where $\mathbf{A}=\left(\mathbf{a}_{i j}\right) \in \operatorname{Mat}(3, R)$ and

$$
\Delta^{\mathbf{I}} \mathbf{x}_{i}=\left(\Delta^{\mathbf{I}} \mathbf{x}_{1}, \Delta^{\mathbf{I}} \mathbf{x}_{2}, \Delta^{\mathbf{I}} \mathbf{x}_{3}\right),
$$

where

$$
\mathbf{x}_{1}=u, \mathbf{x}_{2}=f(u) \cos v, \mathbf{x}_{3}=f(u) \sin v .
$$

The coefficients of the first fundamental form are given by

$$
\left\{\begin{array}{c}
g_{11}=e^{-2 c u}\left(e^{2 c u}+f^{\prime^{2}}(u)\right), \\
g_{22}=e^{-2 c u} f^{2}(u), \\
g_{12}=0 .
\end{array}\right.
$$

If we require $\mathbf{x}(u, v)$ to be conformal, then

$$
e^{2 c u}+f^{\prime^{2}}(u)=f^{2}(u) .
$$

The coefficients of the second fundamental form are given by

$$
\left\{\begin{array}{c}
h_{11}=-\frac{f(u) f^{\prime \prime}(u)}{\sqrt{f^{2}(u)\left(e^{2 c u}+f^{\prime 2}(u)\right)}}, \\
h_{22}=\frac{f^{2}(u)}{\sqrt{f^{2}(u)\left(e^{2 c u}+f^{\prime 2}(u)\right)}} \\
h_{12}=0 .
\end{array}\right.
$$


So the Gaussian curvature $\mathbf{K}$ and the mean curvature $\mathbf{H}$ are calculated by

$$
\mathbf{K}=\frac{-c^{2} f(u)\left(e^{2 c u}+{f^{\prime}}^{2}(u)\right)^{2}-e^{4 c u} f^{\prime \prime}(u)}{f(u)\left(e^{2 c u}+f^{\prime 2}(u)\right)^{2}}
$$

and

$$
\mathbf{H}=\frac{-f(u) f^{\prime \prime}(u)+e^{2 c u}+{f^{\prime}}^{2}(u)}{2 e^{-2 c u}\left(e^{2 c u}+f^{\prime 2}(u)\right) \sqrt{f^{2}(u)\left(e^{2 c u}+f^{\prime 2}(u)\right)}},
$$

respectively. With the conformality condition (3.4), $\mathbf{H}$ is reduced to

$$
\mathbf{H}=\frac{-f^{\prime \prime}(u)+f(u)}{2 e^{-2 c u} f^{3}(u)} .
$$

Let $\mathbf{H}=c$. Then (3.6) can be written as

$$
f^{\prime \prime}(u)-f(u)+2 c e^{-2 c u} f^{3}(u)=0 .
$$

The differential equation (3.7) cannot be solved analytically [15]. If $c=0$, then (3.7) becomes

$$
f^{\prime \prime}(u)-f(u)=0 .
$$

Hence we can see that if $\mathbf{H}=0$ then $c=0$. Thus we have:

Proposition 3.1. Let $\mathbf{M}$ be surfaces of revolution given by (2.6) in $\mathbb{H}^{3}\left(-c^{2}\right)$. If $c \neq 0$, then there are no minimal conformal surfaces of revolution in $\mathbb{H}^{3}\left(-c^{2}\right)$. If $c=0$, then there are minimal conformal surfaces of revolution in $\mathbb{E}^{3}$ if and only if $f^{\prime \prime}-f=0$ which has the general solution $f(u)=c_{1} e^{u}+c_{2} e^{-u}$ for some constant $c_{1}, c_{2}[15]$.

With the conformality condition (3.4), $\mathbf{K}$ is reduced to

$$
\mathbf{K}=\frac{-c^{2} f^{5}(u)-e^{4 c u} f^{\prime \prime}(u)}{f^{5}(u)}
$$

Let $\mathbf{K}=c$. Then (3.8) can be written as

$$
c f^{5}(u)+c^{2} f^{5}(u)+e^{4 c u} f^{\prime \prime}(u)=0 .
$$

The differential equation (3.9) cannot be solved analytically. Hence we see that if $\mathbf{K}=0$ then $c=0$ and $f^{\prime \prime}(u)=0$. Thus we have:

Proposition 3.2. Let $\mathbf{M}$ be surfaces of revolution given by (2.6) in $\mathbb{H}^{3}\left(-c^{2}\right)$. If $c \neq 0$, then there are no flat conformal surfaces of revolution in $\mathbb{H}^{3}\left(-c^{2}\right)$. If $c=0$ then there are flat conformal surfaces of revolution in $\mathbb{E}^{3}$ if and only if $f^{\prime \prime}=0$ which has the general solution $f(u)=c_{1} u+c_{2}$ for some constant $c_{1}, c_{2}$ [15]. 
Assume that $\mathbf{K}=\mathbf{K}_{0} \in \mathbb{R} \backslash\{0\}$. By (3.8), we get

$$
f^{5}(u) \mathbf{K}_{0}+c^{2} f^{5}(u)+e^{4 c u} f^{\prime \prime}(u)=0,
$$

where $f(u) \neq 0$. The differential equation (3.10) cannot be solved analytically for $c \neq 0$. If $c=0$, then the solution of (3.10) given by

$$
f(u)= \pm \frac{3^{\frac{1}{6}} \sqrt{c_{1}^{\frac{1}{3}}\left(-1+J a c o b i C N\left[2.3^{\frac{1}{12}} \sqrt{c_{1}^{\frac{2}{3}}\left(u+c_{2}\right)^{2}\left(-\mathbf{K}_{0}\right)^{\frac{1}{3}}}, \frac{1}{4}(2+\sqrt{3})\right]\right)}}{\sqrt{1+\sqrt{3}} \sqrt{-2+\sqrt{3}-J a c o b i C N\left[2.3^{\frac{1}{12}} \sqrt{c_{1}^{\frac{2}{3}}\left(u+c_{2}\right)^{2}\left(-\mathbf{K}_{0}\right)^{\frac{1}{3}}}, \frac{1}{4}(2+\sqrt{3})\right]}},
$$

where $c_{1}, c_{2} \in \mathbb{R}$.

Theorem 3.1. There is no conformal surface of revolution which has constant the Gaussian curvature in $\mathbb{H}^{3}\left(-c^{2}\right)$. The Gaussian curvature of conformal surface of revolution is constant, $\mathbf{K}=\mathbf{K}_{0}$, in $\mathbb{E}^{3}$ if and only if the function $f(u)$ is (3.11).

Similar calculations are also used for the mean curvature, what we get there is not conformal on the surfaces of revolution which has the real mean curvature in $\mathbb{E}^{3}$. By straightforward computation, the Laplacian operator on $\mathbf{M}$ with the help of (3.1), (3.2), (3.3) and (2.7) turns out to be

$$
\Delta^{\mathbf{I}} \mathbf{X}_{i}=\left(\begin{array}{c}
\frac{e^{2 c u}\left(-f^{\prime}\left(e^{2 c u}+{f^{\prime 2}}^{2}\right)+f\left(c e^{2 c u}+f^{\prime} f^{\prime \prime}\right)\right)}{f\left(e^{2 c u}+f^{\prime 2}\right)^{2}}, \\
\frac{e^{4 c u} \cos v\left(e^{2 c u}+f^{\prime 2}+f\left(c f^{\prime}-f^{\prime \prime}\right)\right)}{f\left(e^{2 c u}+f^{\prime 2}\right)^{2}}, \\
\frac{e^{4 c u} \sin v\left(e^{2 c u}+f^{\prime 2}+f\left(c f^{\prime}-f^{\prime \prime}\right)\right)}{f\left(e^{2 c u}+f^{\prime 2}\right)^{2}}
\end{array}\right) .
$$

With the conformality condition (3.4), the equation (3.12) is reduced to

$$
\Delta^{\mathbf{I}} \mathbf{x}_{i}=\left(\begin{array}{c}
\frac{e^{2 c u}\left(-f^{\prime} f^{2}+f\left(c e^{2 c u}+f^{\prime} f^{\prime \prime}\right)\right)}{f^{5}} \\
\frac{e^{4 c u} \cos v\left(f^{2}+f\left(c f^{\prime}-f^{\prime \prime}\right)\right)}{f^{5}} \\
\frac{e^{4 c u} \sin v\left(f^{2}+f\left(c f^{\prime}-f^{\prime \prime}\right)\right)}{f^{5}}
\end{array}\right) .
$$

Suppose that $\mathbf{M}$ satisfies (3.1). Then from (3.1), we have

$$
\left\{\begin{aligned}
a_{11} u+a_{12} f(u) \cos v+a_{13} f(u) \sin v=\frac{e^{2 c u}\left(-f^{\prime} f^{2}+f\left(c e^{2 c u}+f^{\prime} f^{\prime \prime}\right)\right)}{f^{5}}, \\
a_{21} u+a_{22} f(u) \cos v+a_{23} f(u) \sin v=\frac{e^{4 c u} \cos v\left(f^{2}+f\left(c f^{\prime}-f^{\prime \prime}\right)\right)}{f^{5}} \\
a_{31} u+a_{32} f(u) \cos v+a_{32} f(u) \sin v=\frac{e^{4 c u} \sin v\left(f^{2}+f\left(c f^{\prime}-f^{\prime \prime}\right)\right)}{f^{5}}
\end{aligned}\right.
$$


Since the functions $\cos v, \sin v$ and the constant function are linearly independent, by (3.13) we get $a_{12}=a_{13}=a_{21}=a_{23}=a_{31}=a_{32}=0, a_{11}=\lambda, a_{22}=a_{33}=\mu$. Consequently the matrix $\mathbf{A}$ satisfies

$$
\mathbf{A}=\left[\begin{array}{lll}
\lambda & 0 & 0 \\
0 & \mu & 0 \\
0 & 0 & \mu
\end{array}\right]
$$

The equation (3.13) is rewritten as the following:

$$
\left\{\begin{array}{c}
\lambda u=\frac{e^{2 c u}\left(-f^{\prime} f+c e^{2 c u}+f^{\prime} f^{\prime \prime}\right)}{f^{4}}, \\
\mu f(u)=\frac{e^{4 c u}\left(f+c f^{\prime}-f^{\prime \prime}\right)}{f^{4}} .
\end{array}\right.
$$

This means that $\mathbf{M}$ is at most of 2-type. From (3.14), we have

$$
\left\{\begin{array}{c}
\frac{e^{4 c u}}{f^{4}}=\frac{\lambda u}{c}-\frac{e^{2 c u} f^{\prime}\left(f^{\prime \prime}-f\right)}{c f^{4}}, \\
\frac{e^{4 c u}}{f^{4}}=\frac{\mu f}{f+c f^{\prime}-f^{\prime \prime}} .
\end{array}\right.
$$

Combining the first and the second equation of (3.15), we obtain

$$
\frac{\lambda u}{c}-\frac{e^{2 c u} f^{\prime}\left(f^{\prime \prime}-f\right)}{c f^{4}}=\frac{\mu f}{f+c f^{\prime}-f^{\prime \prime}} .
$$

The equation (3.16) is reduced to

$\left(3.17 \phi^{2 c u} f^{\prime}\left(f-f^{\prime \prime}\right)\left((-1+c) f-f^{\prime \prime}\right)+f^{4}\left(((-1+c) \lambda u+c \mu) f-\lambda u f^{\prime \prime}\right)=0\right.$.

In the cases $\{c \neq 0, \lambda \neq 0, \mu=0\},\{c \neq 0, \lambda=0, \mu \neq 0\},\{c \neq 0, \lambda \neq 0, \mu \neq 0\}$, the second order nonlinear differential equation (3.17) cannot be solved analytically. For the case $\{c \neq 0, \lambda=0, \mu=0\},(3.17)$ can be written as

$$
e^{2 c u} f^{\prime}\left(f-f^{\prime \prime}\right)\left((1-c) f^{\prime}-f^{\prime \prime}\right)=0 .
$$

The general solutions of (3.18) are given by

$$
\left\{\begin{array}{l}
f(u)=c_{1}, \\
f(u)=c_{1} e^{u}+c_{2} e^{-u}, \\
f(u)=c_{1} e^{u \sqrt{c-1}}+c_{2} e^{-u \sqrt{c-1}},
\end{array}\right.
$$

where $c_{1}, c_{2} \in \mathbb{R}$ and $c-1>0$. Substituting the solutions (3.19) into (3.14), respectively. They don' $t$ satisfy these equations. Thus, we can give the following theorem:

Theorem 3.2. Let $\mathbf{M}$ be conformal surfaces of revolution given by (2.6) and $c \neq 0$ in $\mathbb{H}^{3}\left(-c^{2}\right)$. Then there are no harmonic and non-harmonic conformal surfaces of revolution satisfying the conditions $\Delta^{\mathbf{I}} \mathbf{x}=0$ and $\Delta^{\mathbf{I}} \mathbf{x}=A \mathbf{x}$, respectively, where $A \in \operatorname{Mat}(3, \mathbb{R})$. 
In the cases $\{c=0, \lambda \neq 0, \mu \neq 0\},\{c=0, \lambda \neq 0, \mu=0\}$, we cannot obtain any conformal surfaces of revolution in $\mathbb{E}^{3}$. In the cases $\{c=0, \lambda=0, \mu \neq 0\}, \quad\{c=0, \lambda=0, \mu=0\}$, the general solutions of (3.18) are given by

$$
f(u)=c_{1}, f(u)=c_{1} \cos u+c_{2} \sin u,
$$

and

$$
f(u)=c_{1} e^{u}+c_{2} e^{-u},
$$

respectively. The solution $f(u)=c_{1}$ satisfies (3.14) for $\mu=\frac{1}{c_{1}^{4}}$. Then, the parametrization of $\mathbf{M}$ is given by

$$
\mathbf{x}(u, v)=\left(u, c_{1} \cos v, c_{1} \sin v\right) .
$$

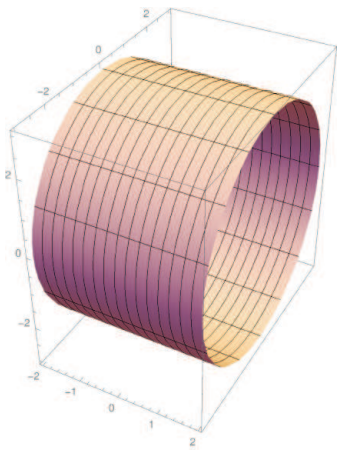

FIG. 3.1:

The solution $f(u)=c_{1} \cos u+c_{2} \sin u$ does not satisfies (3.14). But the solution (3.20) satisfies (3.14). Then, the parametrization of $\mathbf{M}$ is given by

$$
\mathbf{x}(u, v)=\left(u,\left(c_{1} e^{u}+c_{2} e^{-u}\right) \cos v,\left(c_{1} e^{u}+c_{2} e^{-u}\right) \sin v\right) .
$$

Thus we can give the following theorem:

Theorem 3.3. Let $\mathbf{M}$ be conformal surfaces of revolution given by (2.6) and $c=0$ in $\mathbb{E}^{3}$. If $\mathbf{M}$ is harmonic or non-harmonic conformal surfaces of revolution, then it is an open part of the surfaces (3.21) or (3.22), respectively. 


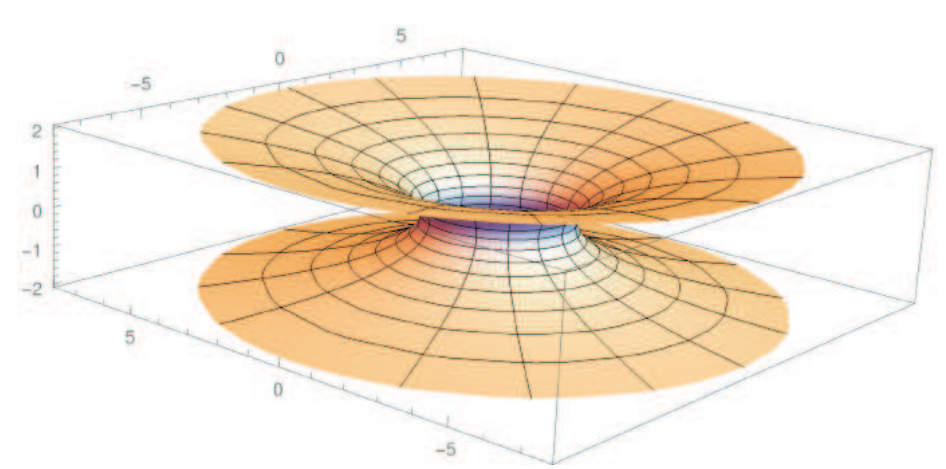

FIG. 3.2:

\section{Conformal Surfaces of Revolution Satisfying $\Delta^{\mathrm{II}} \mathrm{x}=\mathrm{Ax}$}

In this section, we classify conformal surfaces of revolution with non-degenerate second fundamental form in $\mathbb{H}^{3}\left(-c^{2}\right)$ satisfying the equation

$$
\Delta^{\mathbf{I I}} \mathbf{x}=\mathbf{A x}
$$

where $\mathbf{A}=\left(\mathbf{a}_{i j}\right) \in \operatorname{Mat}(3, R)$ and

$$
\Delta^{\mathbf{I I}} \mathbf{x}_{i}=\left(\Delta^{\mathbf{I I}} \mathbf{x}_{1}, \Delta^{\mathbf{I I}} \mathbf{x}_{2}, \Delta^{\mathbf{I I}} \mathbf{x}_{3}\right)
$$

By straightforward computation, the Laplacian operator on $\mathbf{M}$ with the help of (3.2), (3.5), (4.2) and (2.8) turns out to be

$$
\Delta^{\mathbf{I I}} \mathbf{x}_{i}=\left(\begin{array}{c}
\frac{\sqrt{f^{2}\left(e^{2 c u}+f^{\prime 2}\right)}\left(f f^{\prime \prime \prime}-f^{\prime} f^{\prime \prime}\right)}{2 f^{2} f^{\prime 2}{ }^{2}}, \\
\frac{\cos v \sqrt{f^{2}\left(e^{2 c u}+f^{\prime 2}\right)}\left(f f^{\prime} f^{\prime \prime \prime}-f^{\prime 2} f^{\prime \prime}-2 f f^{\prime \prime 2}\right)}{2 f^{2} f^{\prime \prime 2}} \\
\frac{\sin v \sqrt{f^{2}\left(e^{2 c u}+f^{\prime 2}\right)}\left(f f^{\prime} f^{\prime \prime \prime}-f^{\prime 2} f^{\prime \prime}-2 f f^{\prime \prime}\right)}{2 f^{2} f^{\prime \prime}}
\end{array}\right) .
$$

With the conformality condition (3.4), the equation (4.3) is reduced to

$$
\Delta^{\mathbf{I I}} \mathbf{x}_{i}=\left(\begin{array}{c}
\frac{f f^{\prime \prime \prime}-f^{\prime} f^{\prime \prime}}{2 f^{\prime \prime^{2}}} \\
\frac{\cos v\left(f f^{\prime} f^{\prime \prime \prime}-f^{\prime 2} f^{\prime \prime}-2 f f^{\prime \prime 2}\right)}{2 f^{\prime \prime 2}} \\
\frac{\sin v\left(f f^{\prime} f^{\prime \prime \prime}-f^{\prime 2} f^{\prime \prime}-2 f f^{\prime \prime 2}\right)}{2 f^{\prime \prime^{2}}}
\end{array}\right)
$$


Suppose that $\mathbf{M}$ satisfies (4.1). Then from (4.1) and (4.2), we have

$$
\left\{\begin{array}{l}
a_{11} u+a_{12} f(u) \cos v+a_{13} f(u) \sin v=\frac{f f^{\prime \prime \prime}-f^{\prime} f^{\prime \prime}}{2 f^{\prime \prime}} \\
a_{21} u+a_{22} f(u) \cos v+a_{23} f(u) \sin v=\frac{\cos v\left(f f^{\prime} f^{\prime \prime \prime}-f^{\prime 2} f^{\prime \prime}-2 f f^{\prime \prime^{2}}\right)}{2 f^{\prime \prime 2}} \\
a_{31} u+a_{32} f(u) \cos v+a_{32} f(u) \sin v=\frac{\sin v\left(f f^{\prime} f^{\prime \prime \prime}-{f^{\prime}}^{\prime \prime}{\left.f^{\prime \prime}-2 f f^{\prime \prime 2}\right)}_{2 \prime^{2}}\right.}{2 f^{\prime 2}}
\end{array}\right.
$$

Since the functions $\cos v, \sin v$ and the constant function are linearly independent, by (4.4) we get $a_{12}=a_{13}=a_{21}=a_{23}=a_{31}=a_{32}=0, a_{11}=\lambda, a_{22}=a_{33}=\mu$. Consequently the matrix $\mathbf{A}$ satisfies

$$
\mathbf{A}=\left[\begin{array}{lll}
\lambda & 0 & 0 \\
0 & \mu & 0 \\
0 & 0 & \mu
\end{array}\right]
$$

and the equation (4.4) is rewritten as follows:

$$
\left\{\begin{array}{l}
\lambda u=\frac{f f^{\prime \prime \prime}-f^{\prime} f^{\prime \prime}}{2 f^{\prime \prime 2}} \\
\mu f(u) \cos v=\frac{\cos v\left(f f^{\prime} f^{\prime \prime \prime}-f^{\prime 2} f^{\prime \prime}-2 f f^{\prime \prime 2}\right)}{2 f^{\prime \prime 2}{ }^{2}} \\
\mu f(u) \sin v=\frac{\sin v\left(f f^{\prime} f^{\prime \prime \prime}-f^{\prime 2} f^{\prime \prime}-2 f f^{\prime \prime 2}\right)}{2 f^{\prime \prime 2}}
\end{array}\right.
$$

From (4.5), we obtain

$$
\left\{\begin{array}{l}
\lambda u=\frac{f f^{\prime \prime \prime}-f^{\prime} f^{\prime \prime}}{2 f^{\prime \prime 2}}, \\
\mu f(u)=\frac{\left(f f^{\prime} f^{\prime \prime \prime}-f^{\prime 2} f^{\prime \prime}-2 f f^{\prime \prime^{2}}\right)}{2 f^{\prime{ }^{2}}} .
\end{array}\right.
$$

This means that $\mathbf{M}$ is at most of 2-type. From (4.6), we have

$$
\left\{\begin{array}{l}
\frac{f f^{\prime \prime \prime}-f^{\prime} f^{\prime \prime}}{2 f^{\prime \prime 2}}=\lambda u, \\
\frac{f f^{\prime \prime \prime}-f^{\prime} f^{\prime \prime}}{2 f^{\prime \prime 2}}=\frac{f(\mu-1)}{f^{\prime}} .
\end{array}\right.
$$

Combining the first and the second equation of (4.7), we obtain

$$
\lambda u f^{\prime}(u)+f(u)(1-\mu)=0 .
$$

If we solve the ordinary differential equation, we get

$$
f(u)=c_{1} u^{\frac{\mu-1}{\lambda}},
$$

where $\lambda \neq 0, \mu \neq 0, c_{1} \in \mathbb{R}$. If we apply the solution (4.9) into the first and the second line of the equation (4.6), we can easily see that it does not satisfies these equations. If we choose

$$
\lambda=\frac{(\mu-1)^{2}}{\mu-2}, \mu \neq 1, \mu \neq 2,
$$


then the equation (4.8) is reduced to

$$
\left(\frac{(\mu-1)^{2}}{\mu-2}\right) u f^{\prime}(u)+f(u)(1-\mu)=0 .
$$

Its general solution is given by

$$
f(u)=c_{1} u^{\frac{\mu-2}{\mu-1}} .
$$

The solution (4.11) provides the system (4.6). Thus the matrix A satisfies

$$
\mathbf{A}=\left[\begin{array}{ccc}
\frac{(\mu-1)^{2}}{\mu-2} & 0 & 0 \\
0 & \mu & 0 \\
0 & 0 & \mu
\end{array}\right]
$$

Then, the parametrization of $\mathbf{M}$ is given by

$$
\mathbf{x}(u, v)=\left(u, c_{1} u^{\frac{\mu-2}{\mu-1}} \cos v, c_{1} u^{\frac{\mu-2}{\mu-1}} \sin v\right) .
$$

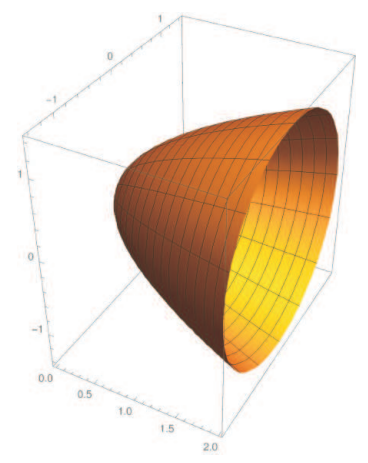

FIG. 4.1:

Let $\mu=0$, then from (4.10), we obtain

$$
f(u)-\frac{u f^{\prime}(u)}{2}=0 .
$$

Its general solution is

$$
f(u)=c_{1} u^{2} .
$$

The solution (4.13) does not satisfies (4.7). Thus we can give the following theorems:

Definition 4.1. A surface in $\mathbb{H}^{3}\left(-c^{2}\right)$ is said to be II-harmonic if it satisfies the condition $\Delta^{\mathrm{II}} \mathbf{X}=\mathbf{0}$. 
Theorem 4.1. Let $\mathbf{M}$ be conformal surfaces of revolution given by (2.6) and $c \neq 0$ in $\mathbb{H}^{3}\left(-c^{2}\right)$. Then there are no II-harmonic conformal surfaces of revolution satisfying the condition $\Delta^{\mathbf{I I}} \mathbf{x}=0$.

Theorem 4.2. Let $\mathbf{M}$ be conformal surfaces of revolution given by (2.6) and $c \neq 0$ in $\mathbb{H}^{3}\left(-c^{2}\right)$. If the surface $\mathbf{M}$ satisfies the condition $\Delta^{\mathbf{I I}} \mathbf{x}=$ Ax , where $A \in$ Mat $(3, \mathbb{R})$, then it is an open part of the surface (4.12).

\section{Conformal Surfaces of Revolution Satisfying $\Delta^{\mathrm{III}} \mathrm{x}=\mathbf{A x}$}

In this section, we will classify conformal surfaces of revolution with non-degenerate second fundamental form in $\mathbb{H}^{3}\left(-c^{2}\right)$ satisfying the equation

$$
\Delta^{\mathrm{III}} \mathrm{x}=\mathbf{A x},
$$

where $\mathbf{A}=\left(\mathbf{a}_{i j}\right) \in \operatorname{Mat}(3, R)$ and

$$
\Delta^{\mathbf{I I I}} \mathbf{x}_{i}=\left(\Delta^{\mathbf{I I I}} \mathbf{x}_{1}, \Delta^{\mathbf{I I I}} \mathbf{x}_{2}, \Delta^{\mathbf{I I I}} \mathbf{x}_{3}\right) .
$$

By straightforward computation, the Laplacian operator on $\mathbf{M}$ with the help of (3.2), (3.3), (3.5), (5.2) and (2.9) turns out to be

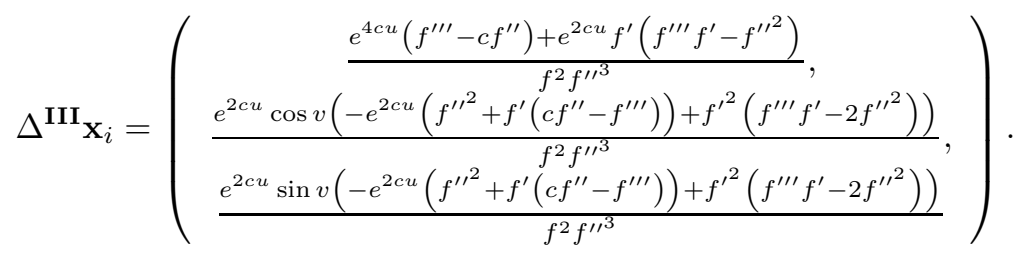

Suppose that $\mathbf{M}$ satisfies (5.1). Then from (5.1) and (5.2), we have

$$
\begin{gathered}
a_{11} u+a_{12} f(u) \cos v+a_{13} f(u) \sin v=A(u) e^{2 c u} \\
a_{21} u+a_{22} f(u) \cos v+a_{23} f(u) \sin v=B(u) e^{2 c u} \cos v \\
a_{31} u+a_{32} f(u) \cos v+a_{33} f(u) \sin v=B(u) e^{2 c u} \sin v,
\end{gathered}
$$

where

$$
\begin{aligned}
& A(u)=\left(\frac{e^{2 c u}\left(f^{\prime \prime \prime}-c f^{\prime \prime}\right)+f^{\prime}\left(f^{\prime \prime \prime} f^{\prime}-f^{\prime \prime^{2}}\right)}{f^{2} f^{\prime \prime^{3}}}\right), \\
& B(u)=\frac{\left(-e^{2 c u}\left(f^{\prime \prime^{2}}+f^{\prime}\left(c f^{\prime \prime}-f^{\prime \prime \prime}\right)\right)+f^{\prime^{2}}\left(f^{\prime \prime \prime} f^{\prime}-2 f^{\prime \prime^{2}}\right)\right)}{f^{2} f^{\prime \prime^{3}}} .
\end{aligned}
$$

Since the functions $\cos v, \sin v$ and the constant function are linearly independent, by (5.4) we get $a_{12}=a_{13}=a_{21}=a_{23}=a_{31}=a_{32}=0, a_{11}=\lambda, a_{22}=a_{33}=\mu$. 
Consequently the matrix A satisfies

$$
\mathbf{A}=\left[\begin{array}{lll}
\lambda & 0 & 0 \\
0 & \mu & 0 \\
0 & 0 & \mu
\end{array}\right]
$$

and (5.4) is rewritten as the following:

$$
\begin{gathered}
\lambda u=\frac{e^{4 c u}\left(f^{\prime \prime \prime}-c f^{\prime \prime}\right)+e^{2 c u} f^{\prime}\left(f^{\prime \prime \prime} f^{\prime}-f^{\prime^{2}}\right)}{f^{2} f^{\prime^{3}}}, \\
\mu f(u)=\frac{e^{2 c u}\left(-e^{2 c u}\left(f^{\prime \prime^{2}}+f^{\prime}\left(c f^{\prime \prime}-f^{\prime \prime \prime}\right)\right)+f^{\prime^{2}}\left(f^{\prime \prime \prime} f^{\prime}-2 f^{\prime \prime^{2}}\right)\right)}{f^{2} f^{\prime \prime^{3}}} .
\end{gathered}
$$

Combining the first and the second equation of (5.5), we obtain

$$
e^{4 c u}+e^{2 c u} f^{\prime 2}+\mu f^{3} f^{\prime \prime}-u \lambda f^{2} f^{\prime} f^{\prime \prime}=0
$$

In the cases $\{c \neq 0, \lambda \neq 0, \mu=0\},\{c \neq 0, \lambda=0, \mu \neq 0\},\{c \neq 0, \lambda \neq 0, \mu \neq 0\}$ and $\{c=0, \lambda \neq 0, \mu=0\},\{c=0, \lambda=0, \mu \neq 0\},\{c=0, \lambda \neq 0, \mu \neq 0\}$, we can not obtain any conformal surfaces of revolution in $\mathbb{H}^{3}\left(-c^{2}\right)$ and $\mathbb{E}^{3}$, respectively. Because the second order nonlinear differential equation (5.6) cannot be solved analytically. We will discuss two cases according to constant $c$.

Case 1: Let $c \neq 0, \lambda=0, \mu=0$, from (5.6), we obtain

$$
e^{4 c u}+e^{2 c u} f^{\prime 2}=0 .
$$

Its general solution is

$$
f(u)=c_{1} \pm i \frac{e^{c u}}{c}
$$

Case 2: Let $c=0, \lambda=0, \mu=0$, from (5.6), we obtain

$$
1+f^{\prime^{2}}=0
$$

The general solution of the equation (5.9) is given by

$$
f(u)=c_{1} \pm i u
$$

Since the solutions (5.8) and (5.10) are complex, it is a contradiction. Thus we can give the following theorem:

Definition 5.1. A surface in $\mathbb{H}^{3}\left(-c^{2}\right)$ is said to be III-harmonic if it satisfies the condition $\Delta^{\mathbf{I I I}} \mathbf{x}=\mathbf{0}$. 
Theorem 5.1. Let $\mathbf{M}$ be conformal surfaces of revolution given by (2.6) and $c \neq$ 0 in $\mathbb{H}^{3}\left(-c^{2}\right)$. Then there are no III-harmonic and non III-harmonic conformal surfaces of revolution satisfying the conditions $\Delta^{\mathbf{I I I}} \mathbf{x}=0$ and $\Delta^{\mathbf{I I I}} \mathbf{x}=A \mathbf{x}$, where $A \in \operatorname{Mat}(3, \mathbb{R})$, respectively.

Theorem 5.2. Let $\mathbf{M}$ be conformal surfaces of revolution given by (2.6) and $c=0$ in $\mathbb{E}^{3}$. Then there are no III-harmonic and non III-harmonic conformal surfaces of revolution satisfying the conditions $\Delta^{\mathbf{I I I}} \mathbf{x}=0$ and $\Delta^{\mathbf{I I I}} \mathbf{x}=A \mathbf{x}$, where $A \in M a t$ $(3, \mathbb{R})$, respectively.

\section{Acknowledgment}

This paper was supported by Erciyes University Coordinatorship of Scientific Research Project Unit (ProjectNo: FBA-2017-7364).

\section{REF E R E N C E S}

1. E. Abbena, S. Salamon and A. Gray: Modern Differential Geometry of Curves and Surfaces with Mathematica. CRC Press. 92 (2006).

2. L. J. Alias, A. Ferrandez and P. Lucas: Surfaces in the 3-dimensional LorentzMinkowski space satisfying $\Delta x=A x+B$. Pacific J. Math. 156 (1992), 201-208.

3. C. Baikoussis and L. Verstraelen: On the Gauss map of helicoidal surfaces. Rend. Sem. Math. Messina Ser. II 2(16) (1993), 31-42.

4. M. Bekkar and H. Zoubir: Surfaces of Revolution in the 3-Dimensional LorentzMinkowski Space Satisfying $\Delta x^{i}=\lambda^{i} x^{i}$. Int. J. Contemp. Math. Sciences. 3(24) (2008), $1173-1185$.

5. S. M. CHOI: On the Gauss map of surfaces of revolution in a 3-dimensional Minkowski space. Tsukuba J. Math. 19 (1995), 351-367.

6. M. Choi, Y. H. Kim and D. W. Yoon: Some classification of surfaces of revolution in Minkowski 3-space. J. Geom. 104 (2013), 85-106.

7. .B Y. Chen: A report on submanifold of finite type. Soochow J. Math. 22 (1996), $117-337$.

8. F. Dillen, J. Pas and L. Vertraelen: On surfaces of finite type in Euclidean 3-space. Kodai Math. J. 13 (1990), 10-21.

9. F. Dillen, J. Pas and L. Vertraelen: On the Gauss map of surfaces of revolution. Bull. Inst. Math. Acad. Sinica. 18 (1990), 239-246.

10. O. J. GaraY: $n$ extension of Takahashi's theorem. Geom. Dedicata. 34 (1990), 105112.

11. E. Guler and O. KISI: The Second Laplace-Beltrami Operator on Rotational Hypersurfaces in the Euclidean 4-Space. Mathematica Aeterna. 8(1) (2018), 1-12.

12. G. Kaimakamis, B. Papantoniou and K Petoumenos: Surfaces of revolution in the 3-dimensional Lorentz-Minkowski space satisfying $\Delta^{I I I} r=A r$. Bull.Greek Math. Soc. 50 (2005), 75-90. 
13. E. P. LAMBERT: Lorentz invariant spacelike surfaces of constant mean curvature in anti de sitter 3-space. Msc. Thesis, The University of Southern Mississippi, 2015.

14. S. LEE: Spacelike surfaces of constant mean curvature one in de Sitter 3-space. Illinois Journal of Mathematics. 49(1) (2005), 63-98.

15. S. LEE and K. ZARSKE : Surfaces of revolution with constant mean curvature in Hyperbolic 3-space. Differential Geometry - Dynamical Systems (DGDS). 16 (2014), 203-218.

16. S. LEE and J. MARTIN : Timelike surfaces of revolution with constant mean curvature in de sitter 3-space. International Electronic Journal of Geometry (IEJG). 8 (1) (2015), 116-127.

17. B. Senoussi and M. BekKAR : Helicoidal surfaces with $\Delta^{J} r=A r$ in 3-dimensional Euclidean space. Stud. Univ. Babes-Bolyai Math. 60(3) (2015), 437-448.

18. T. TAKAhashi : Minimal immersions of Riemannian manifolds. J. Math. Soc. Japan. 18 (1996), 380-385.

19. J. VRŠEK and M. LÁVIČKA: Determining surfaces of revolution from their implicit equations. Journal of Computational and Applied Mathematics. 290 (2015), 125-135.

\author{
Nural Yuksel \\ Erciyes University \\ Faculty of Science \\ Department of Mathematics \\ 38030 Kayseri, Turkey \\ yukseln@erciyes.edu.tr \\ Murat Kemal Karacan \\ Uşak University \\ Faculty of Sciences and Arts \\ Department of Mathematics \\ 64200 Uşak, Turkey \\ murat.karacan@usak. edu.tr
}

